\title{
Variations of the Coronal Radiation in X-ray Related to Coronal Holes, Active Region Loop Systems, Bright Points
}

\author{
By ESTER ANTONUCCI
}

Istituto di Fisica Generale, University of Torino, 10125 Torino, Italy

\begin{abstract}
The coronal features observed in X-ray emission, varying from the small-scale, short-lived bright points to the large-scale, long-lived coronal holes, are closely associated with the coronal magnetic field and its topology, and their variability depends strongly on the solar cycle. Here we discuss the spatial distribution of the coronal structures, the frequency distribution of the brightness variations in active regions, and the role of magnetic reconnection in determining the variability of the coronal features, on the basis of the new observations of the soft X-ray emission recently obtained with the Yohkoh satellite and the NIXT experiment.
\end{abstract}

\section{Introduction}

The highly variable character of the solar X-ray emission through the solar cycle and the tendency to observe an increasing variability with photon energy became immediately evident since the first X-ray observations performed in the fifties. The X-ray emission appeared to be not only variable in time but also highly inhomogeneous: the first $\mathrm{X}$ ray image of the Sun obtained on April 19, 1960, by the Naval Research Laboratory researchers, showed that approximately $80 \%$ of the emission was coming from $5 \%$ or less of the area of the disk.

Only with the Skylab experiments, in the early 70's, however, were the characteristics of the highly variable nature and the detailed structure of the corona definitely established. Coronal loops were recognized as the basic elements of the solar corona (Rosner et al. 1978a), since the X-ray emission comes principally from coronal regions of closed magnetic fields, while it becomes negligible in coronal holes where the field is believed to be open. A wealth of interconnections and interactions between the different coronal regions was discovered. It was also possible to establish the existence in the transition region and in the corona of structures down to a few arcseconds, that is down to the instrumental resolution.

An extremely important discovery, during Skylab, was that magnetic fields are not only acting to confine the solar plasma in the corona, but they are also playing a fundamental role in coronal heating. Rosner et al. $(1978 \mathrm{~b})$ pointed out that two elements are very likely necessary to produce a hot corona: a relatively strong magnetic field coupled with an input of mechanical energy into the field. At approximately the same time, Athay \& White $(1978 ; 1979)$ were establishing on the basis of the OSO VIII observations, that the acoustic waves originating in the photosphere do not represent an effective agent for coronal heating, being stopped before reaching the corona, thus supporting the view of a coronal heating process strictly related to the presence of the magnetic field.

The picture of the solar corona emerging from Skylab and the OSO satellites was that of an inhomogeneous atmosphere, consisting of features of different scales, each with a different role in different phases of the solar cycle. Active regions are intermediate structures, dominant in the rising phase of the cycle and at solar maximum, when with the beginning of the new cycle the relatively simple configuration of the magnetic field typical of solar minimum is disrupted by the emergence of the new toroidal field. Active 
regions originate non-uniformly over the solar disk, and they are typically organized in latitude belts migrating toward the equator. Coronal holes, reflecting the long-lived large-scale pattern of the solar magnetic fields, are dominant in the declining phase of solar activity, when the solar magnetic field is dominated by the lower order multipoles. They appear as large structures extending over 1-5\% of the solar disk, with a fast growth, followed by a relatively slow evolution, and an overall lifetime of several solar rotations. In the declining phase, coronal holes are typically longitudinal structures extending from low latitudes to the poles. At solar minimum the X-ray bright points (XBP's), which appear as small ( $\leq 1$ arcmin), short-lived ( $\leq 2$ days) active regions, are the dominant structures, with a rather uniform distribution on the solar disk (Golub et al. 1979).

The overall picture of the solar corona emerging with Skylab remained substantially unchanged till the late 80 's, when a new potentiality for coronal studies was provided by achieving the capability of subarcsecond imaging in the extreme ultraviolet and soft X-rays, and with the launch in 1991 of the Japanese satellite, Yohkoh. The Soft X-ray Telescope (SXT) on Yohkoh is almost continuously imaging the solar corona in the region from $2 \AA$ to $40 \AA$ with a wide dynamic range and an excellent spatial resolution (2.5 arcsec pixels). The almost continuous monitoring of the Sun provided by Yohkoh is revealing in detail the extremely dynamic behavior of the corona, which is in continuous evolution from the small to the large-scale. The sub-arcsecond imaging of the solar corona obtained with NIXT, made possible by the advent of telescopes carrying multilayercoated optics responding in the XUV range, has revealed a complex coronal fine structure down to $600 \mathrm{~km}$, the instrument resolution (Golub et al. 1990).

\section{Spatial distribution of coronal structures in active regions}

According to the picture emerging from Skylab observations, the large majority of the material of an active region above the chromosphere is emitting at a temperature above $10^{6}{ }^{\circ} \mathrm{K}$. The emission comes mainly from compact hot loops, $10^{4}-10^{5} \mathrm{~km}$ in length scale, with their footpoints in bright plages of opposite magnetic polarity, sites for strong fields. Hot bright loops, visible in hotter ions such as $\mathrm{Mg}$ IX, are not the only constituents of an active region. They coexist with cool loops which are visible in cooler ions formed below $10^{6}{ }^{\circ} \mathrm{K}$, such as Ne VII, and they are typically thinner, longer, less stable and less numerous than hot loops. Hot and cool loops are structures forming in different parts of an active region; hot loops are clustered across the neutral line, while the cooler longer loops are rooted in the periphery of active regions (Webb 1981).

The coronal portion of the loop is inferred to be quasi-isothermal while the cool emission is confined near the footpoints due to the high conductivity of the solar plasma which tends to smooth the temperature variations along the loop. Typical quiescent regions appear to be formed by individual plasma loops whose temperatures cluster in a narrow range within 2 and $3 \times 10^{6}{ }^{\circ} \mathrm{K}$ and with a temperature gradient at the loop base inferred to be above $2 \times 10^{-2}{ }^{\circ} \mathrm{K} \mathrm{cm}^{-1}$.

The largest brightness variations between the loops, or within the same loop, are principally due to density rather than temperature variations. Neupert (1968) suggested chromospheric evaporation as the most likely explanation for density enhancements in the solar corona. That is, flows of plasma from the dense chromosphere to the rarefied corona can be induced whenever during transient energy release phenomena only a negligible amount of the energy deposited in the upper chromosphere can be dissipated. In this case, the energy in excess is transferred to the corona in form of an upward enthalpy flux. 
The NIXT experiment, initiated successfully with the rocket launched on September 11,1989 , provided sub-arcsecond $(0.75$ arcsec $)$ narrow band images at $63.5 \AA$, which include the emission from the Fe XVI and $\mathrm{Mg} \mathrm{X}$ ions (Golub et al. 1990). NIXT observations clearly evidenced the existence of fine structure, down to the instrumental resolution, at all locations in the corona, showing that the magnetic complexity observed in the photosphere is probably reflected at coronal levels (Golub et al. 1990). These observations are strongly suggesting that coronal loops are further structured into even more elementary features.

A possible filamentary nature of the corona was previously suggested through indirect evidence derived from the Solar Maximum Mission (SMM) observations, which led to infer a width of the filaments of the order of 100-300 km (Linford \& Wolfson 1988; Martens et al. 1985). In fact the density of the coronal plasma derived by direct methods, such as by using density-sensitive line ratios, is usually found to exceed the density inferred by combining the plasma emission measure and the scale of the soft X-ray emission region. This can be interpreted as an indication of the non-uniform distribution of the plasma in the coronal region which is concentrated in filamentary structures, not yet resolved in the observations.

When studying the scale distribution of the coronal structures with NIXT images, which resolve magnetic features down to about $600 \mathrm{~km}$, Gomez et al. (1993a) conclude that no preferred length-scale can be found for X-ray coronal structures in active regions. The two-dimensional power spectra on the wavenumber plane are substantially isotropic with no significant individual peaks as one would expect if active regions were consisting of a number of magnetic fluxtubes of given size with no internal structures. In this case, in fact, power spectra would exhibit peaks corresponding to the characteristic size of the fluxtubes.

The omnidirectional spectra, obtained by calculating the average spectrum in rings of increasing radius, are power law with the same power law index $\alpha$ constant for the different active regions. This conclusion suggests that the process generating the $\mathrm{X}$ ray emission is substantially the same for active regions. Dark regions behave in a qualitatively different manner and do not share the same power law distribution found for active regions. NIXT results are confirmed by a similar analysis performed on the Yohkoh images (Martens \& Gomez 1992), which however yields a power law index, $\alpha=-2.4$, which is somewhat different from the NIXT value, $\alpha=-3$ (Gomez et al. 1993a). The difference, if interpreted in the light of the different responses of the instruments, would imply that hotter structures tend to be smaller in size, the Yohkoh soft X-ray telescope SXT being more sensitive to higher temperatures.

Gomez et al. (1993b) have been able to model the observed spatial distribution of the $\mathrm{X}$-ray emission, by assuming that the Poynting flux entering the corona is proportional to the radiative losses in the coronal plasma and by relating it to the fluctuating velocity and magnetic fields in the photosphere which can be assumed to be also distributed according to a power law.

\section{Temporal variability in active regions}

\subsection{Transient brightenings}

Emission in active regions is highly variable not only during the most energetic phenomena such as flares and subflares, but also when active regions are in their 'quiescent' conditions. The amplitude of the brightness variations in active regions is much larger in the less frequent and more energetic events and decreases with increasing frequency 
of the variation. According to the Skylab observations (e.g. Sheeley 1980; Webb 1981), obtained in years of declining activity, with typical time resolution within 1.5 minutes to 3 hours, in an active region in 'quiescent conditions' the cooler emission from the legs of the bright hot loops was typically changing on time scales of minutes, while the brightness of the hotter coronal portion of the loop was found to be constant over periods comparable to the radiative lifetime (Gerassimenko et al. 1978). Individual loops were found to evolve on a time scale of several hours, while the overall pattern might persist for several days. This fact was interpreted as evidence for steady-state heating.

The recent results obtained on an almost continuous basis with the SXT on Yohkoh reveal in active regions a high variability also on a short term, with frequent brightenings in compact loops. 'Active' active regions, as observed in the years of maximum activity, show a coronal variability of the order of $10^{2} \mathrm{~s}$ within the overall active region configuration which evolves on a much longer time scale of $10^{5} \mathrm{~s}$.

Transient brightenings indicating episodes of energy release in compact loops in the low corona, with lengthscale from a few $10^{8} \mathrm{~cm}$ to $5 \times 10^{9} \mathrm{~cm}$, localized in regions of 10 arcseconds in size in the hottest portions of the loops, occur with a timescale of about 3 minutes and tend to be recurrent (Shimizu et al. 1992). A longer time scale, about 1 hour, is observed for the occurrence of transient brightenings in 'quieter' active regions. A typical transient brightening is characterized by a temperature of $7 \times 10^{6}{ }^{\circ} \mathrm{K}$ and an emission measure of about $7 \times 10^{28} \mathrm{~cm}^{-5}$, by a factor of 1.3 and 6 larger than in quiet conditions, respectively, an electron density approximately doubled, of $10^{10} \mathrm{~cm}^{-3}$, and an energy release of $5 \times 10^{28}$ ergs (Shimizu et al. 1992), of the same order of magnitude of the energy associated with microflares. Interestingly enough, the total soft X-ray flux of an active region is found to be correlated with the frequency of occurrence of the transient brightenings in the active region.

These new findings confirm the existence of small-scale high-temperature $\left(\approx 10^{7}{ }^{\circ} \mathrm{K}\right)$ regions in non-flaring active regions discovered during SMM (Martens et al. 1985), and point to the episodic nature of the heating process in active regions. Heating by discrete events was proposed on a theoretical ground in a series of papers by Parker, who has introduced the concept of nanoflares, consisting in localized releases of magnetic energy due to small-scale magnetic reconnection events in the process of topological dissipation (e.g. Parker 1988). The energy released in a transient brightening is however much larger than that predicted for nanoflares $\left(\ll 10^{26}-10^{27} \mathrm{ergs}\right)$.

\subsection{Variability related to flares}

The occurrence rate of transient brightenings in active regions as a function of the total energy released in the brightening has not yet been established. This relation is instead well established for the more energetic transient brightness variations in active regions which form the large class of flare events: the rate of occurrence of the brightness variations in active regions is decreasing with increasing amplitude of the variation, that is with the rate of energy release.

The observations of microflares in hard X-rays (13-600 keV) by Lin et al. (1984) confirmed the tendency for less energetic events to occur more frequently. Microflares are also observed in the UV emission (e.g., C IV, $\lambda=1548 \AA, T=10^{5}{ }^{\circ} \mathrm{K}$ ) which is increasing of $30 \%-100 \%$ on a time scale of $10-60 \mathrm{sec}$, with an energy release rate of $10^{7} \mathrm{erg} \mathrm{s}^{-1} \mathrm{~cm}^{-2}$, two orders of magnitude larger than in quiescent active regions (Porter et al. 1984, 1987; Porter 1992). The fluctuating component represents about $60 \%$ of the total UV emission in years of maximum activity and is also correlated with the soft X-ray $(3.5-5.5 \mathrm{keV})$ variability.

The Solar Maximum Mission detection in hard X-rays of thousands of flares collected 
over a decade of observations (1980-1989) has allowed a very accurate determination of the distribution of the flare occurrence rate. Hard X-ray flares are distributed over four orders of magnitude in peak flux, ranging from microflares at the limit of instrumental sensitivity to events which are so intense to saturate the detectors flown up to now.

All of the relevant parameters characterizing the energy release as observed in hard $\mathrm{X}$ rays (25-500 keV) can be described by a power law distribution $d N / d x \propto x^{-\alpha}$ (Crosby et al. 1993). These SMM results confirm previous findings obtained by investigating the hard X-ray (Datlowe et al. 1974; Lin et al. 1984) and soft X-ray flare emission (1-2 keV) (e.g., Drake 1971) as well as the interplanetary particle events. The power law index remains fairly constant for the different flare parameters, $1.4 \leq \alpha \leq 2$; a flatter distribution is however found for interplanetary particle events, $1.1 \leq \alpha \leq 1.3$ (Van Hollebeke et al. 1975; Cliver et al. 1991). The slope of the frequency distribution of UV microflares is also consistent with the values found for hard X-ray microflares.

By combining the integral rate of flare occurrence for the data sets obtained by Datlowe et al. (1974) with OSO-VII and by Crosby et al. (1993) with the Solar Maximum Mission, and of microflares obtained during the rocket flights by Lin et al. $(1984,1991)$, it can be shown that while the flare frequency can vary considerably between the solar cycle maximum and minimum, the slope of the frequency distribution remains substantially invariant, although fluctuating on a yearly basis.

The frequency distribution of flare energy is fully consistent with the model developed by Rosner \& Vaiana (1978), which assumes an exponential growth for the flare energy build up, followed by a stochastic flaring, when all the free energy built up between two consecutive flares is released. The slope of the frequency distribution in this model can be related to the characteristic energy build up time, to the average time between flares as well as to the average dissipated energy per flare. The quasi-invariance through the solar cycle of the slope of the flare frequency distribution implies that the average energy dissipation rate per flare and the magnetic build up time remain approximately constant through the solar cycle. Hence, the solar cycle variability of the flaring rate (which can vary by a factor of 20 ) is suggested to be principally caused by a variation in the number of flaring active regions more than in the average flaring rate per active region (Crosby et al. 1993).

The power law distribution for flare occurrence is also found to be consistent with avalanche models. The hypothesis at the basis of these models is that the magnetic energy released by reconnection at a given location is transported to the nearby sites inducing further reconnection. A simulation where magnetic stress is injected periodically at random sites yields a power law with slope consistent with the observations (Lu \& Hamilton 1991). Zirker \& Cleveland (1993) introducing in the avalanche model specific physical mechanisms causing the energy release have been capable to reproduce a frequency distribution which might correspond to the nanoflare distribution with energy per event up to $10^{26} \mathrm{ergs}$, by twisting the coronal loop footpoints by granular velocity, and the flare distribution up to $10^{28}-10^{29}$ ergs, by braiding of neighboring loops.

When integrating the flare distribution over flare energy within $10^{26}-10^{32} \mathrm{ergs}$, the total dissipated power in the solar corona is of the order of $10^{25} \mathrm{erg} \mathrm{s}^{-1}$. This quantity is insufficient to explain the solar soft X-ray luminosity of $7 \times 10^{27} \mathrm{erg} \mathrm{s}^{-1}$ (Mewe 1972). These considerations on the energetics of the corona has led Hudson (1991) to invoke a separate. soft X-ray distribution for episodic events, such as nanoflares, in order to support the idea of general coronal heating sustained by episodic events proposed by Parker (1988). 


\subsection{Coronal variability during flares}

During the individual events of flare energy release no significant periodic variability has been found, except in very few cases: for example, a $1.6 \mathrm{~s}$ periodicity was observed for as long as 700 cycles in soft X-rays during the August 7, 1972 flare (Thomas et al. 1987). Flares are, however, often characterized by a quasi-periodic variability which can be observed at different wavelengths. Characteristic time delays between quasi-periodic events are typically within $1.6 \mathrm{~s}$ and $36 \mathrm{~s}$ in microwave, hard X-ray and $\gamma$ emission (e.g., Kiplinger et al. 1983; Kane et al. 1983; Nakajima et al. 1983; Forrest \& Chupp 1983; Desai et al. 1987; Kosugi \& Kiplinger 1987). Characteristic rise and decay times of $10 \mathrm{~s}$ are found for the soft X-ray emission fluctuations (Zarro et al. 1987), and fluctuations above $20 \mathrm{~s}$ are observed in the UV, namely, in the Fe XXI emission line (Cheng 1987). The quasi-periodic variability during flares on time scales of $1-10 \mathrm{~s}$ might be interpreted as consistent with the concept introduced by de Jager and de Jonge (1978) that the flare energy is released in a large number of elementary flare processes, releasing $10^{27}-10^{28}$ ergs. Quantities of energy of the same order can be easily stored in an elementary flux tube due to the photospheric twisting motions of the footpoints (e.g. Sturrock et al. 1984).

Episodic energy release below or of the order of $10^{28} \mathrm{ergs}$ in sporadic events is continually present on the Sun in form of hard X-ray microflares (Lin et al. 1984), soft X-ray brightenings (Shimizu et al. 1992), and UV microflares (Porter 1992). It remains to be established whether a flare can be produced by an integration of several small-scale localized heating processes (as in the case proposed by Parker 1987, in which heating occurs for dissipation of magnetic energy at several small-scale tangential discontinuities scattered throughout the region interested in the energy release), as suggested to explain coronal heating, or whether, to account for the high flare energy release rates $\geq 10^{29} \mathrm{erg} \mathrm{s}^{-1}$, the magnetic dissipation has to occur predominantly on larger scales (e.g. determined by the dimension of the surface separating magnetic fluxes of opposite polarities where magnetic reconnection can occur). If the agent of energy release is indeed magnetic reconnection, the question is then whether in a flare the energy is released preferentially by reconnecting current sheets on a scale size of the order of the flare region or in numerous elementary current sheets scattered throughout the flare region.

The high temporal resolution observations of the hard X-ray emission during the SMM period have also definitely established the existence of a subsecond variability, first observed by Hoyng et al. (1976). Sub-second fluctuations are observed in hard X-rays by Kiplinger et al. (1983), Hurley et al. (1983), Bai (1987), and in microwaves, at frequencies of $22-44 \mathrm{GHz}$, by Kaufmann et al. (1984). The most plausible origin of the hard X-ray variability on a sub-second timescale can be found in rapid changes in the production of non-thermal electrons. Microbursts on time-scales of $10-10^{2} \mathrm{~ms}$ have been suggested to be related to the magnetic islands developing in a current sheet during magnetic reconnection (Sturrock 1987).

\section{Coronal hole evolution}

Coronal holes, defined as the sites where the soft X-ray emission is drastically reduced, have been studied in detail during Skylab. They are dominant on the solar disk in the years preceding solar minimum, when the magnetic dipole is well-established. At this time their size is more extended and their lifetime is more prolonged. They develop according to the pattern of the large-scale coronal magnetic fields and they are associated with the large unipolar magnetic regions of open field lines as inferred by the extrapola- 
tion of the surface fields (Levine et al. 1977). Coronal holes first appear at low latitudes, and then tend to connect with the poles of the same polarity. Following a rapid growth, they evolve slowly and eventually shrink and disappear. Active regions can drift across the coronal hole without substantial alteration of that pattern.

\subsection{Coronal hole rotation}

One aspect which confirms the close connection of coronal holes to the large-scale magnetic fields in the corona is the rotational behavior of the coronal holes. Both coronal holes (Timothy et al. 1975; Wagner 1975) and the large-scale coronal magnetic pattern (Antonucci \& Svalgaard 1974; Hoeksema \& Scherrer 1987) are not significantly sheared by differential rotation, and this phenomenon is more evident when activity is declining. Furthermore, the north-south asymmetry found by Antonucci et al. (1990) for the large-scale photospheric field rotation during cycle 21 (that is the northern hemisphere is rotating faster, 26.9 days, than the southern hemisphere, 28.1 days) is consistent with the eastward migration of coronal holes, following a 28.5 days pattern in the south and a 27 days one in the north observed by Sheeley \& Harvey (1981). The coronal hole study was performed by Sheeley and Harvey by using the He I (10830 $\AA$ ) emission as a proxy of the soft X-ray emission, not available during the solar maximum of cycle 21 .

Direct systematic investigations of coronal holes in soft X-rays have been resumed only recently. The SXT/ Yohkoh observations have established that the rigid rotation of individual soft $\mathrm{X}$-ray coronal holes is not only a characteristic of the declining phase of activity, but it is preserved throughout the solar cycle. Thin long coronal holes are observed with the SXT to rotate at a rate close to the equatorial one during solar maximum (Tsuneta \& Lemen 1993).

\subsection{Evolution of coronal hole boundaries}

The investigation of the evolution of coronal hole boundaries is essential in order to understand the coronal hole rotation properties. Skylab observations led to the determination that the average areal changes, $d A / d t=1.510^{4} \mathrm{~km}^{2} \mathrm{~s}^{-1}$, are consistent with the diffusion by supergranular cells (Bohlin 1977; Nolte et al. 1978a). Transient changes of coronal hole boundaries were also put in relation to the occurrence of X-ray coronal transients and filament eruptions (Webb et al. 1978; Rust 1983). Evidence for a statistical relationship between the X-ray bright point density and the rate of contraction of coronal holes was put forward by Nolte et al. (1978b), but also evidence against a simple relationship between XBP's and coronal holes was found (Davis 1985), therefore this relationship remained in doubt.

A recent re-examination of the Skylab observations of the extended, long-lived coronal hole CH 1 by Kahler \& Moses (1990), however, has established a scale of boundary changes, $1.7 \times 10^{4} \mathrm{~km}$, below the supergranular cell size, leading to exclude a relationship between the evolution of coronal hole boundaries and supergranular diffusion. This same analysis suggests that X-ray bright points are responsible both for contractions and expansions of $\mathrm{CH} 1$, and the boundary variations associated with bright points are contributing in such a way to preserve rigid rotation of the coronal hole pattern, being predominantly eastward below $20^{\circ}$ and predominantly westward above $20^{\circ}$ in latitude. Kahler \& Moses (1990) are therefore suggesting that the closed magnetic fields of bright points and the open field lines in coronal holes reconnect at coronal hole boundaries in such a way to maintain the rigid rotation behavior. According to this interpretation, therefore, no systematic flux emergence is required to maintain rigid rotation.

The well-established rotational properties of coronal holes and this new evidence for the role played by magnetic reconnection in contributing to maintain the coronal hole 
boundaries unsheared by differential rotation are substantially consistent with a model of coronal magnetic fields developed by Nash et al. (1988). The corona is assumed as a current-free region within the source surface for the interplanetary magnetic field (as defined by Schatten et al. 1969). The coronal field is determined as follows. Its radial component is matching the photospheric flux distribution, which is differentially rotating, while its non-radial component has to be equal to zero at the source surface. Coronal holes being the footpoint locations of open field lines, are connected to the source surface where the lower order multipoles tend to dominate. Therefore they share the rotational properties of the lower order multipoles and then rotate quasi rigidly. The hole is allowed to follow the source surface field through reconnection of the magnetic field lines. This process makes it possible to uncouple the motion of the hole boundaries from that of the photospheric fields sheared by differential rotation.

According to the model by Nash et al. (1988), then, large-scale magnetic fields are not deriving their fluxes from the dispersal of active regions, but their pattern is an intrinsic property of the organization in multipoles of the solar magnetic field. Polar holes with meridional extensions and prolonged rigid rotation, as observed during the Skylab period in the declining phase of cycle 20 , are expected on the basis of this model when a strong axisymmetric field component is assumed to be dominant. When, on the other hand, non-axisymmetric field components dominate, a less-rigid rotation is expected for coronal holes.

\subsection{Mass fiux in coronal holes}

An analysis of C IV profiles, obtained with the High Resolution Telescope and Spectrograph (HRTS), confirms that downflows are observed at all locations of the solar atmosphere in the transition region; however there is a greater proportion of outflows in coronal holes than in the quiet Sun (Dere et al. 1989). That is, in coronal holes there are more regions than in the quiet Sun characterized by outflows. The average outflow velocity in coronal hole regions is $5 \mathrm{~km} \mathrm{~s}^{-1}$, while the average downflow velocity is within 5 and $12 \mathrm{~km} \mathrm{~s}^{-1}$ in the quiet Sun and decreases to $2 \mathrm{~km} \mathrm{~s}^{-1}$ in coronal holes. On the other hand, the average non-thermal velocities are in both cases comparable, $\sim 28 \mathrm{~km}$ $\mathrm{s}^{-1}$ (Doschek et al. 1976; Roussel-Dupré \& Shine 1982; Dere et al. 1984, 1989).

Assuming only $1 \%$ of the solar surface covered by coronal holes, with a transition region density of $10^{10} \mathrm{~cm}^{-3}$, the observed outflow velocities yield a solar wind mass flux of $1.310^{15}$ protons $\mathrm{cm}^{-2} \mathrm{~s}^{-1}$ (Dere et al. 1989), to be compared with a proton flux of about $10^{14}$ protons $\mathrm{cm}^{-2} \mathrm{~s}^{-1}$ inferred at the base of the solar wind streams (e.g. Zirker 1976). The HRTS observations are then strongly supporting the current idea of coronal holes as source of the fast streams in the solar wind.

A surprising result comes from the recent Yohkoh observations which have been used to establish a mass loss in the solar wind coming from the outward expansion of the coronal active regions (Uchida et al. 1992). This might be a relevant observational evidence, in view of a possible identification of the source of the low-speed solar wind. The active region outward expansion is conjectured to be related to the occurrence of brightenings in active regions. For a single active region expansion, Uchida et al. (1992) have determined a rate of mass loss of $10^{10}-10^{11} \mathrm{~g} \mathrm{~s}^{-1}$, assuming coronal densities of $10^{9} \mathrm{~cm}^{-3}$, and a magnetic flux loss of $10^{16.5} \mathrm{Mx} \mathrm{s}^{-1}$.

\section{X-ray bright points}

X-ray bright points were first studied in detail during Skylab (Golub et al. 1974, 1977). They are uniformly distributed on the solar disk and they have been associated 
with elementary bipolar magnetic regions. The He I $10830 \AA$ line has usually been assumed as a proxy in the bright point studies performed after Shylab, due to the lack of direct soft X-ray emission observations.

With the discovery of bright points it became clear that the size of active regions on the Sun could be considered as forming a continuous distribution, where the number of active regions is a monotonically decreasing function of either size or lifetime (Golub 1980 ). Bright points were considered at the extreme of the size distribution of the regions observed in soft $\mathrm{X}$-rays, being defined as elementary active regions with dimensions below 60 arcsec (Webb 1981).

Owing to the significant improvement in instrumental resolution, the loop structure of $\mathrm{X}$-ray bright points, also inferred from the association of XBP's with magnetic bipolar regions, has been better resolved in the NIXT observations (Golub et al. 1990). The investigation of the NIXT images has led to identify a number of X-ray bright points which is below the expectation on the basis of the Golub's empirical law, which predicts a cyclic variation in the XBP number in anticorrelation with the active region cycle.

The systematic study of soft X-ray bright points, as for coronal holes, has been resumed with the SXT experiment on Yohkoh (Strong et al. 1992). According to a recent investigation of XBP's (Harvey et al. 1993), only $30 \%$ of magnetic bipoles coincide with XBP's, but the majority of XBP's are associated with bipolar magnetic areas of decreasing magnetic flux, with a decrease exceeding on the average $310^{14} \mathrm{Mx} \mathrm{s}^{-1}$, in absolute value. However, the association with regions where the magnetic flux is decreasing is neither a necessary nor a sufficient condition for the occurrence of XBP's (Harvey et al. 1993). XBP's are rather associated with chance encounters of features of opposite magnetic polarity, conclusion that was also reached by considering the $\mathrm{He} I$ dark points.

Large amplitude fluctuations in the soft X-ray emission of bright points, within $30 \%$ and $200 \%$, have characteristic time scales of a few minutes and are superimposed to a slower evolution in the emission (Strong et al. 1992). The largest fluctuations are defined as XBP flares. A similar variability was found for EUV bright points by Habbal $\&$ Withbroe (1981). The observations strongly suggest that the XBP variability can be related to magnetic reconnection of features of opposite polarity. The occurrence of magnetic reconnection is further supported by an excellent observation of a plasma jet at a velocity of $1000 \mathrm{~km} \mathrm{~s}^{-1}$, associated with a bright point flare which was observed to be related to magnetic flux emergence (Harvey et al. 1993).

The rate of XBP's emergence observed with the SXT is exceeding that expected from Golub's empirical law, result which is inconsistent with the NIXT results. The responses of NIXT and SXT to temperature are however different, and this might be at the basis of this apparent contradiction.

\section{Summary}

New results on the spatial and temporal distributions of the soft X-ray emission have been obtained recently with the SXT telescope on Yohkoh, which is monitoring on an almost continuous basis the solar corona at high resolution, and with the introduction of sub-arcsecond imaging of the soft X-ray corona with the NIXT experiment.

The corona is extremely structured also on the small-scale down to $600 \mathrm{~km}$, the best present instrumental resolution. Possible structuring on still finer scales is suggested on the basis of the NIXT observations and the density measurements obtained with SMM. No preferred scale sizes are observed in active regions and the slope of the size distribution is approximately invariant.

Frequent transient brightenings are observed in active region loops which may support 
the concept of a corona heated by a large number of elementary energy release processes. The distribution of the flare occurrence rate is characterized by a slope which remains approximately constant during a solar cycle, implying that the average energy dissipation rate per flare remains constant through the cycle, while the number of flaring active regions is considerably varying.

Very likely the dissipation of magnetic energy responsible for the release of energy observed in the coronal fluxtubes, in moderately active conditions as well as in flares, occurs through magnetic reconnection, a process which might also be in general responsible for the evolution of coronal features. In this connection, reconnection of magnetic field lines is suggested to be the most probable candidate to explain the evolution of the coronal hole boundaries, and also to play an essential role in the variability of the $\mathrm{X}$-ray emission of bright points.

\section{REFERENCES}

Antonucci, E. \& SvalgaARD, L. 1974 Rigid and differential rotation of the solar corona. Solar Physics 34, 3-10.

Antonucci, E., Hoeksema, J. T. \& Scherrer, P.H. 1990 Rotation of the photospheric magnetic fields: a north-south asymmetry. The Astrophysical Journal 360, 296-304.

AтнAY, R. G. \& White, O. R. 1978 Chromospheric and coronal heating by sound waves. The Astrophysical Journal 226, 1135-1139.

Athay, R. G. \& White, O. R. 1979 Chromospheric oscillations observed with OSO 8. II. Average power spectra for Si II. The Astrophysical Journal Suppl. 39, 333-346.

BAI, T. 1987 Sub-second variations of high-energy $(\geq 300 \mathrm{keV})$ hard X-ray emission from solar flares. In Rapid Fluctuations in Solar Flares NASA CP-2449, 29-33.

BoHLIN, J.D. 1977 Extreme-ultraviolet observations of coronal holes. I: Locations, sizes and evolution of coronal holes, June 1973 - January 1974. Solar Physics 51, 377-398.

Cheng, C.-C. 1987 A search for fast variations in the Fe XXI emission during solar flares. In Rapid Fluctuations in Solar Flares NASA CP-2449, 263-269.

Cliver, E., Reames, D., Kahler, S. \& Cane H. 1991 Size distribution of solar energetic particle events. Proc. 22nd Int. Cosmic Ray Conf. 3, 25-28.

Crosby, N. B., Aschwanden, M. J. \& Dennis B. R. 1993 Frequency distributions and correlations of soft X-ray flare parameters. Solar Physics 143, 275-299.

Datlowe, D. W., Elcan, M. J., \& Hudson, H. S. 1974 OSO-7 observations of solar X-rays in the energy range $10-100 \mathrm{keV}$. Solar Physics 39, 155-174.

Davis, J. M. 1985 Small-scale flux emergence and the evolution of equatorial coronal holes. Solar Physics 95, 73-82.

DE JAGer, C. \& DE JoNGe G. 1978 Properties of elementary flare bursts. Solar Physics 58, 127-137.

Dere, K. P., Bartoe, J.-D. F. \& Brueckner, G. E. 1984 High-resolution telescope and spectrograph observations of the quiet solar chromosphere and transition zone. The Astrophysical Journal 281, 870-883.

Dere, K. P., Bartoe, J.-D. F., Brueckner, G. E. \& Recely, F. 1989 Transition zone flows observed in a coronal hole on the solar disk. The Astrophysical Journal 345, L95-L97.

Desai, U. D., Kouveliotou, C., Barat, C., Hurley, K., Niel, M., Talon, R. \& VeDRENNE G. 1987 Persistent time intervals between features in solar flare hard X-ray emission. In Rapid Fluctuations in Solar Flares NASA CP-2449, 63-74.

Doschek, G. A., Feldman, U. \& Bohlin, J. D. 1976 Doppler wavelength shifts of transition zone lines measured in Skylab solar spectra. The Astrophysical Journal 205, L177-L180.

Drake, J.F. 1971 Characteristics of soft solar X-ray bursts. Solar Physics 16, 152-185.

Forrest, D. J. \& ChUPP, E. L. 1983 Simultaneous acceleration of electrons and ions in solar flares. Nature 305, 291-292. 
Gerassimenko, M., Solodyna, C. V. \& Nolte J. T. 1978 Observational evidence of continual heating in X-ray emitting coronal loops. Solar Physics 57, 103-110.

Golub, L. 1980 X-ray bright points and the solar cycle. Proc. Roy. Soc. A. 297, 595-604.

Golub, L., Krieger, A. S., Silk, J. K., Timothy, A. F. \& Vaiana, G. S. 1974 Solar X-ray bright points. The Astrophysical Journal 189, L93-L97.

Golub, L., Krieger, A. S., Vaiana, G. S. \& Harvey, J. W. 1977 Magnetic properties of X-ray bright points. Solar Physics 53, 111-121.

Golub, L., Davis, J. M. \& KrIEger, A. S. 1979 Anticorrelation of X-ray bright points with sunspot number, 1970 - 1978. The Astrophysical Journal 229, L145-L150.

Golub, L., ET AL. 1990 Subarcsecond observations of the solar X-ray corona. Nature 344, $842-844$.

Gomez, D. O., Martens, P. C. H. \& Golub, L. 1993a Normal incidence X-ray telescope power spectra of X-ray emission from solar active regions. I. Observations. The Astrophysical Journal 405, 767-772.

Gomez, D. O., Martens, P. C. H. \& Golub, L. 1993b Normal incidence X-ray telescope power spectra of X-ray emission. II. Theory. The Astrophysical Journal 405, 773-781.

HabBal, S. R. \& Withbroe, G. L. 1981 Spatial and temporal variations of EUV coronal bright points. Solar Physics 69, 77-97.

Harvey, K. L., Nitta, N., Strong, K. T. \& Tsuneta, S. 1993 The relationship of X-ray bright points to the photospheric magnetic fields. In preparation.

Hoyng, P., Brown J. C. \& VAN BeEk, H. F. 1976 High time resolution analysis of solar hard X-ray flares observed on board the ESRO TD-1A satellite. Solar Physics 48, 197-254.

Hoeksema, J. T. \& Scherrer, P. H. 1987 Rotation of the coronal magnetic field. The Astrophysical Journal 318, 428-436.

Hudson, H. S. 1991 Solar flares, microflares, nanoflares, and coronal heating. Solar Physics 133, 357-369.

Hurley, K., Niel, M., Talon, R., Estulin, I. V. \& Dolidze V.Ch. 1983 Multispacecraft hard X-ray observations of fine time structure in two solar flares. The Astrophysical Journal 265, 1076-1983.

Kahler, S. W. \& Moses, D. 1990 Discrete changes in solar coronal hole boundaries. The Astrophysical Journal 362, 728-733.

Kane, S. R., Kai, K., Kosugi, T., Enome, S., Landecker, P. B. \& McKenzie, D. L. 1983 Acceleration and confinement of energetic particles in the 1980 June 7 solar flare. The Astrophysical Journal 271, 376-387.

Kaufmann, P., Correia, E., Costa, J. E. R., Dennis, B. R., Hurford, G. J. \& Brown, J. C. 1984 Multiple energetic injections in a strong spike-like solar burst. Solar Physics $\mathbf{9 1}$, $359-376$.

Kiplinger, A. L., Dennis, B. R., Frost, K. J. \& Orwig, L. E. 1983 Recurrent pulse trains in the solar hard X-ray flare of 1980 June 7. The Astrophysical Journal 273, 783-794.

Kosugi, T. \& Kiplinger, A. L. 1987 Quasi-periodic pulsations in solar hard X-ray and microwave flares. In Rapid Fluctuations in Solar Flares NASA CP-2449, 185-191.

Levine, R. H., Altschuler, M. D., Harvey, J. W. \& Jackson, B. V. 1977 Open magnetic structures on the Sun. The Astrophysical Journal 215, 636-651.

Lin, R. P., Schwartz, R. A., Kane, S. R., Pelling, R. M. \& Hurley, K. C. 1984 Solar hard X-ray microflares. The Astrophysical Journal 283, 421-425.

Lin, R. P., Hurley, K. C., Smith, D. M. \& Pelling, R. M. 1991 A search for hard X-ray , microflares near solar minimum. Solar Physics 135, 57-64.

LINFORD, G. A. \& Wolfson, C. J. 1988 Properties of an impulsive compact solar flare determined from Solar Maximum Mission X-ray measurements. The Astrophysical Journal 331, 1036-1046.

Lu, E. T. \& Hamilon, R. J. 1991 Avalanches and the distribution of solar flares. The Astrophysical Journal 380, L89-L92. 
Martens, P. C. H., Van den Oord, G. H. J. \& Hoyng, P. 1985 Observations of steady anomalous magnetic heating in thin current sheets. Solar Physics 96, 253-275.

Martens, P. C. H. \& Gomez, D. O. 1992 Spatial power-spectra from Yohkoh soft X-ray images. PASJ 44, L187-L191.

Mewe, R. 1972 Calculated solar X-ray radiation from 1 to $60 \AA$. Solar Physics 22, 459-491.

NaKajima, H., Kosugi, T., KaI, K. \& ENome, S. 1983 Successive electron and ion accelerations in impulsive solar flares on 7 and 21 June 1980. Nature 305, 292-294.

Nash, A. G., Sheeley, N. R., JR. \& WANG, Y.-M. 1988 Mechanisms for rigid rotation of coronal holes. Solar Physics 117, 359-389.

Neupert, W. M. 1968 Comparison of solar X-ray line emission with microwave emission during flares. The Astrophysical Journal 153, L59-L64.

Nolte, J. T., Krieger, A. S. \& Solodyna, C. V. 1978a Short term evolution of coronal hole boundaries. Solar Physics 57, 129-139.

Nolte, J. T., Davis, J. M., Gerassimenko, M., Krieger, A. S., Solodyna, C. V. \& GoLUB, L. 1978b The relationship between solar activity and coronal hole evolution. Solar Physics 60, 143-153.

PARKER, E. N. 1987 Stimulated dissipation of magnetic discontinuities and the origin of solar flares. Solar Physics 111, 297-308.

PARKer, E. N. 1988 Nanoflares and the solar X-ray corona. The Astrophysical Journal 330, 474-479.

PorTer, J. G. 1992 Microflares observed with UVSP and HXIS. In Coronal Streamers, Coronal Loops, and Coronal and Solar Wind Composition ESA, SP, 348, 289-292.

Porter, J. G., Toomre, J. \& GebBie, K. B. 1984 Frequent ultraviolet brightenings observed in a solar active region with Solar Maximum Mission. The Astrophysical Journal 283, 879-886.

Porter, J. G., Moore, R. L., Reichmann, E. J., Engvold, O. \& Harvey, K. L. 1987 Microflares in the solar magnetic network. The Astrophysical Journal 323, 380-390.

ROSNER, R. \& VAIANA, G. S. 1978 Cosmic flare transients: constraints upon models for energy storage and release derived from the event frequency distribution. The Astrophysical Journal 222, 1104-1108.

Rosner, R., Tucker, W. H. \& Vaiana, G. S. 1978a Dynamics of the quiescent solar corona. The Astrophysical Journal 220, 643-665.

Rosner, R., Golub, L., Coppi, B. \& Vaiana, G. S. 1978b Heating of coronal plasma by anomalous current dissipation. The Astrophysical Journal 222, 317-332.

Roussel-Dupré, D. \& Shine, R. A. 1982 Evidence of redshifts in the average solar line profiles of C IV ans Si IV from OSO-8 observations. Solar Physics 77, 329-340.

Rust, D. M. 1983 Coronal disturbances and their terrestrial effects. Space Sci. Rev. 34, 21-36.

Schatten, K. H., Wilcox, J. M. \& Ness N. F. 1969 A model of interplanetary and coronal magnetic fields. Solar Physics 6, 442-455.

SheEley, N. R., JR. 1980 Temporal variations of loop structures in the solar atmosphere. Solar Physics 66, 79-87.

Sheeley, N. R., JR. \& HaRVEY, J. W. 1981 Coronal holes, solar wind streams, and geomagnetic disturbances during 1978 and 1979. Solar Physics 70, 237-249.

Shimizu, T., Tsuneta, S., Acton, L. W., Lemen, J. R. \& Uchida, Y. 1992 Transient brightenings in active regions observed by the Soft X-ray Telescope on Yohkoh. PASJ 44, L147-L153.

Strong, K. T., Harvey, K. L., Hirayama, T., Nitta, N., Shimizu, T. \& Tsuneta, S. 1992 Observations of the variability of coronal bright points by the Soft X-ray Telescope on Yohkoh. PASJ 44, L161-L166.

STURRock, P. A. 1987 Rapid fluctuations in solar flares. In Rapid Fluctuations in Solar Flares NASA CP-2449, 1-28.

Sturrock, P. A., Kaufmann, P., Moore, R. L. \& Smith, D. F. 1984 Energy release in 
solar flares. Solar Physics 94, 341-357.

Timothy, A. F., Krieger, A. S. \& VaianA, G. S. 1975 The structure and evolution of coronal holes. Solar Physics 42, 135-156.

Thomas, R. J., Neupert, W. M. \& Thompson, W. T. 1987 Soft X-ray oscillations during the flare of 7 August 1972. In Rapid Fluctuations in Solar Flares NASA CP-2449, 299-300.

Tsuneta, S. \& Lemen J. R. 1993 Dynamics of the solar corona observed with the Yohkoh Soft X-ray Telescope. In Physics of Solar and Stellar Coronae: G.S. Vaiana Memorial Symposium (eds. J. F. Linsky and S. Serio). pp. 113-130. Kluwer Academic Publishers.

Uchida, Y., McAllister, A., Strong, K. T., Ogawara, Y., Shimizu, T., Matsumoto, R. \& Hudson, H. S. 1992 Continual expansion of the active-region corona observed by the Yohkoh Soft X-ray Telescope. PASJ 44, L155-L160.

VAN Hollebeke, M. A. I., MA Sung, L. S. \& McDonald, F. B. 1975 The variation of solar proton energy spectra and size distribution with heliolongitude. Solar Physics 41, 189-223.

WAGNER, W. J. 1975 Solar rotation as marked by extreme-ultraviolet coronal holes. The Astrophysical Journal 198, L141-L144.

WEBB, D. F. 1981 Active region structures in the transition region and corona. In Solar Active Regions. A Monograph from Skylab Solar Workshop III (ed. F.Q. Orrall). pp. 165-198. Colorado Associated University Press, Boulder, CO., USA.

Webb, D. F., McIntosh, P. S., Nolte, J. T. \& Solodyna, C. V. 1978 Evidence linking coronal transients to the evolution of coronal holes. Solar Physics 58, 389-396.

ZARro, D. M., SABA, J. L. R. \& Strong, K. T. 1987 Rapid soft X-ray fluctuations in solar flares observed with the X-ray Polychromator. In Rapid Fluctuations in Solar Flares NASA CP-2449, 289-298.

ZIRKER, J. B. 1976 Energy balance in coronal holes and solar wind streams. In The Energy Balance and Hydrodynamics of the Solar Chromosphere and Corona (ed. R.-M. Bonnet \& P. Delache). 421-445.

Zirker, J. B. \& Cleveland, F. M. 1993 Avalanche models of active region heating and flaring. Solar Physics 145, 119-128. 